\title{
Effect of tidal triggering on seismicity in Taiwan revealed by the empirical mode decomposition method
}

\author{
H.-J. Chen ${ }^{1}$, C.-C. Chen ${ }^{1}$, C.-Y. Tseng ${ }^{2}$, and J.-H. Wang ${ }^{3}$ \\ ${ }^{1}$ Department of Earth Sciences and Graduate Institute of Geophysics, National Central University, Jhongli, Taiwan \\ ${ }^{2}$ Department of Oncology, University of Alberta, Edmonton, AB T6G 1Z2, Canada \\ ${ }^{3}$ Institute of Earth Sciences, Academia Sinica, Nangang, Taipei, 115, Taiwan
}

Correspondence to: C.-C. Chen (chencc@earth.ncu.edu.tw)

Received: 22 December 2011 - Revised: 31 March 2012 - Accepted: 10 May 2012 - Published: 12 July 2012

\begin{abstract}
The effect of tidal triggering on earthquake occurrence has been controversial for many years. This study considered earthquakes that occurred near Taiwan between 1973 and 2008. Because earthquake data are nonlinear and non-stationary, we applied the empirical mode decomposition (EMD) method to analyze the temporal variations in the number of daily earthquakes to investigate the effect of tidal triggering. We compared the results obtained from the nondeclustered catalog with those from two kinds of declustered catalogs and discuss the aftershock effect on the EMD-based analysis. We also investigated stacking the data based on inphase phenomena of theoretical Earth tides with statistical significance tests. Our results show that the effects of tidal triggering, particularly the lunar tidal effect, can be extracted from the raw seismicity data using the approach proposed here. Our results suggest that the lunar tidal force is likely a factor in the triggering of earthquakes.
\end{abstract}

\section{Introduction}

The effect of tides on the triggering of earthquakes has been widely investigated since the 1930s (Nasu et al., 1931; Davison, 1934; Stetson, 1935; Allen, 1936; Knopoff, 1964; Simpson, 1967; Tsuruoka et al., 1995; Vidale et al., 1998; Souchay and Stavinschi, 1999; Tanaka et al., 2002; Cochran et al., 2004). Allen (1936) tabulated earthquakes into different groups according to the lunar-hour angle of the moon and observed a relationship between the occurrence of earthquakes and the biweekly oscillation of tides. Souchay and Stavinschi (1999) used two statistical methods adopted in Kilston and Knopoff (1983) and a binomial distribution to investigate the correlations between long period tides (>several days) and earthquake occurrence and indicated that earthquakes occur preferentially during the ascending part of the oscillations with periods of $18.2 \mathrm{yr}, 182.62$ days and 13.66 days (i.e. the fortnightly tide). Cochran et al. (2004) compared earthquake focal mechanisms with tidal stress and showed a correlation between strong tides and the occurrence of shallow thrust earthquakes.

As for the Taiwan area, Lin et al. (2003) converted the occurrence times of earthquakes in Taiwan between 1973 and 1991 to lunar time to study the possible correlation between earthquake occurrence and lunar time. They observed significant clustering of smaller events $\left(2.5 \leqq M_{\mathrm{L}} \leqq 5\right)$ around day 14 and day 27 of each lunar month and a $30 \%$ higher earthquake frequency around the day of the full moon than other days. They concluded that the lunar tide can indeed trigger some small earthquakes, while there is no tidal triggering effect for earthquakes of $M_{\mathrm{L}}>5$ and $M_{\mathrm{L}}<2.5$. Additionally, Wang and Kuo (1998) found a significant Poisson component in the frequency distribution of interoccurrence times of $M_{\mathrm{L}} \geqq 7$ earthquakes in the Taiwan region, which suggests that the tidal effect is not significant for triggering $M_{\mathrm{L}} \geqq 7$ earthquakes in this region. Those studies (Wang and Kuo, 1998; Lin et al., 2003) indicate that it is difficult to determine the tidal triggering effect when only examining earthquakes of $M_{\mathrm{L}}>5$.

Several recent studies (Lambert et al., 2009; Métivier et al., 2009; Tanaka, 2010) have confirmed the existence and complex nature of the tidal triggering effect. Nevertheless, whether the Earth tide is a significant triggering factor is still debated (Knopoff, 1964; Simpson, 1967; Heaton, 1982; Vidale et al., 1998). We argue that two limitations of the 
methodologies used in previous investigations affect their analyses. First, several previous studies focused only on earthquakes with magnitudes larger than 5 (Simpson, 1967; Tsuruoka et al., 1995; Tanaka et al., 2002), which might prevent significant information about the tidal triggering effect from being extracted from the data. Second, some of the analyses were based on limited data sets or localized samples (Allen, 1936; Knopoff, 1964; Palumbo, 1986; Vidale et al., 1998). Insufficient data would decrease the reliability of the statistics (Huang, 2006), leading to inconclusive results. In the Taiwan region, the Philippine Sea Plate is subducting underneath the Eurasian Plate (Tsai et al., 1977; Wu, 1978). This collision between the two plates causes high levels of seismicity (Wang, 1988, 1998) (see Fig. 1), which aids in studying the effect of tidal triggering on seismicity in this region.

The purpose of this study was to revisit the relationship between Earth tides and earthquake occurrence. Earth tides, which are primarily caused by gravity from the moon and the sun, usually cause a periodic variation of crustal stresses, with peak amplitudes on the order of $1 \mathrm{kPa}$. The stress change is much smaller than the tens of MPa that is typical of stress drops in earthquakes (cf. Kanamori and Anderson, 1975). The rate of change of tidal stress, however, is greater than the rate of increase of tectonic stress. A small tidal stress could therefore trigger an earthquake when the stress on a fault is near its failure limit. Hence, when the periodic tidal stress reaches the peak, the total stress exerted on the fault could be higher than the stress threshold on the fault. This would induce higher seismicity on days with peak tidal stress than other days; in other words, there is likely to be a positive correlation between tidal stress and earthquake occurrence. Keilis-Borok (1990) argued that numerous physical processes transform the lithosphere into a large nonlinear system that is instable and chaotic. The tidal stress is one of these nonlinear physical processes. Hence, earthquakes that result from the tectonic stress combined with the tidal stress must be nonlinear and nonstationary.

Huang et al. (1998) proposed the Hilbert-Huang transform method, which is based on empirical mode decomposition (EMD), to analyze nonlinear and/or non-stationary data. This method can be applied to analyze the nonlinear and non-stationary time series of earthquakes. Note that the EMD method is designed to adaptively decompose time series into several intrinsic mode functions (IMFs). The method proposed here consists of two steps. The first step is signal separation from earthquake occurrence data through EMD. The possible triggering sources can be identified in the IMFs. Second, the Fourier transform (FT) is used instead of the Hilbert transform to calculate the power spectral density of each IMF. In addition, the temporal variation in the theoretical Earth tides can be considered as an inherent clock for stacking the raw data to increase the signal-to-noise $(\mathrm{S} / \mathrm{N})$ ratio, because the raw data usually consist of both useful signals and background noise.

\section{Taiwan Seismicity}

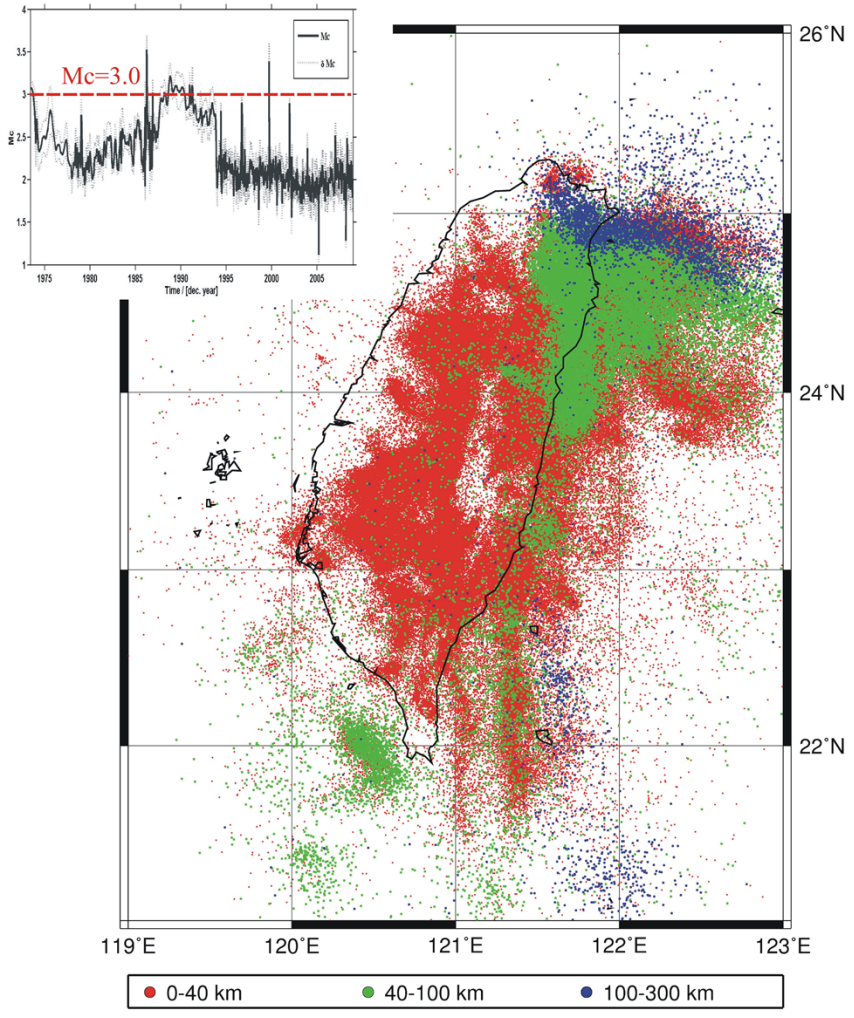

Fig. 1. Spatial distribution of earthquake epicenters with different depths highlighted by color (red dots for $0-40 \mathrm{~km}$, green for 40 $100 \mathrm{~km}$, and blue for $100-300 \mathrm{~km}$ ) and the temporal variation in magnitude of completeness $\left(M_{\mathrm{c}}\right)$ in the Taiwan region.

In this study, we will examine the effect of tides on triggering earthquakes in the Taiwan region based on the IMFs decomposed from the time series of daily earthquake numbers by the EMD method. Because the regional data set consists of several tens of thousands of events, sample size will not be an issue in the statistical analysis.

\section{Data}

The Taiwan Telemetered Seismographic Network (TTSN) (Wang, 1989) was operated by the Institute of Earth Sciences (IES), Academia Sinica, from 1973 to 1992. The network consists of 24 stations, each equipped with a vertical high-gain and analog velocity seismometer. The Taiwan Seismic Network (TSN), operated by the Central Weather Bureau (CWB) of Taiwan, has been upgraded from the old CWB seismic network since 1991, and many new stations have been constructed. In 1992, the TTSN was merged into the TSN. A detailed description of the TSN can be found in Shin (1992). At present, the TSN is composed of 72 stations, each equipped with three-component digital velocity seismometers. This network provides high-quality digital 
seismograms that are recorded in both high- and low-gain formats.

The $M_{\mathrm{L}} \geqq 0$ earthquakes that occurred from 1973 to 2008 are taken from the database provided by the CWB. Figure 1 illustrates the spatial distribution of epicenters, with different depths highlighted by color. Figure 1 also shows the temporal variation of the magnitude of completeness $\left(M_{\mathrm{c}}\right)$ in the non-declustered earthquake catalog (Wiemer and Wyss, 2000). The $M_{\mathrm{c}}$ is the minimum magnitude in the frequency-magnitude distribution (FMD) that fits the Gutenberg-Richter law (Gutenberg and Richter, 1954). Given the temporal variation in $M_{\mathrm{c}}$ in the Taiwan region from 1973 to 2008 , we only consider events with $M_{\mathrm{L}} \geqq M_{\mathrm{c}}=3.0$. A total of 63280 events in the non-declustered catalog are used in this study, which is at least three times larger than the number of events used in other studies (e.g. Tsuruoka et al., 1995; Vidale, 1998). To consider the possible effects of aftershocks, and because the declustering technique is open and non-unique (Huang, 2008), we removed clustered events using both the Resenberg (denoted by R) (Resenberg, 1985) and the Gardner and Knopoff (denoted by GK) (Gardner and Knopoff, 1974) algorithms. The parameters used in the R-declustering algorithm are as follows: $\tau_{\min }=1, \tau_{\max }=$ $10, P_{1}=0.95, X_{k}=0.5, X_{\mathrm{MEFF}}=1.5$, and $\mathrm{R}_{\mathrm{fact}}=10$. The parameters used in the GK-declustering algorithm are the same special-temporal windows as those used in $\mathrm{Wu}$ and Chen (2007). The FMDs of the R- and GK-declustered earthquake catalogs with event magnitudes larger than $M_{\mathrm{c}}=3.0$ could also fit the Gutenberg-Richter law. A total of 43573 and $23505 \mathrm{R}$ - and GK-declustered earthquakes were used, which were $\sim 69 \%$ and $\sim 37 \%$ of the non-declustered cata$\log$, respectively.

\section{Methods}

This section describes the proposed analysis strategy, which consists of five techniques, including source separation using EMD, entropic analysis, spectral analysis using the FT, data stacking, and statistical significance tests.

\subsection{Source separation using EMD}

First, we applied the EMD method to decompose the time series into several components, which are associated with the possible sources (or mechanisms) of triggering earthquakes. As mentioned above, the underlying mechanisms of triggering earthquakes are either nonlinear or non-stationary. The EMD method has been shown to be adaptive and especially efficient for analyzing nonlinear and non-stationary signals (Huang et al., 1998). The method consists of a sifting process to eliminate riding waves on a nonlinear and nonstationary signal, making the wave profiles as symmetric as possible. This process results in a series of intrinsic oscillatory modes or intrinsic mode functions (IMFs) with different instantaneous frequencies. An IMF satisfies two conditions: (1) The numbers of the extrema and zero crossings in the data set must either be equal or differ by at most one datum; and (2) the mean values of the envelope that is formed from the local maxima or the local minima are null at any point. Because IMFs are adaptive and locally determined, they are physically representative of the underlying mechanisms (Huang et al., 1998; Kizhner et al., 2004). The decomposed IMFs are orthogonal to one another and thus can be used as the basis to represent the data.

The sifting process is briefly described below. Suppose that a real signal $S_{\text {real }}(t)$ is contaminated by noise $n(t)$ as $S_{\text {raw }}(t)=S_{\text {real }}(t)+n(t)$. Let $U(t)$ and $L(t)$ be the upper and lower envelopes of $S_{\text {raw }}(t)$, respectively, which are formed by the local maxima and minima of $S_{\text {raw }}(t)$. The sifting process first determines a mean of $U(t)$ and $L(t)$, i.e. $m_{1}(t)=\frac{U(t)+L(t)}{2}$. Subtracting $m_{1}(t)$ from $S_{\text {raw }}(t)$ yields the first IMF component $h_{1}(t)$, i.e. $h_{1}(t)=S_{\text {raw }}(t)-m_{1}(t)$. However, there might be overshoots and undershoots in creating the two envelopes from the real data. Furthermore, the extrapolation used to connect the two extrema can result in an error at the end point. Thus, the sifting process needs to be iterated several times to $h_{1 k}(t)=h_{1(k-1)}(t)-m_{1 k}(t)$, where $k$ denotes the number of iterations until $C_{1}(t)=h_{1 k}(t)$ is an IMF. The function $C_{1}(t)$ is the first IMF with the highest instantaneous frequency and can be separated from $S_{\text {raw }}(t)$ by $\mathrm{R}_{1}(t)=S_{\text {raw }}(t)-C_{1}(t)$.

The sifting process is again conducted on the residual $\mathrm{R}_{1}(t)$ to extract the second IMF and so on until either the residual is less than a given value, giving a meaningful result, or the residual becomes a monotonic function. In principle, $S_{\text {raw }}(t)$ is the superposition of all given IMFs plus the residual $\mathrm{R}_{N}(t)$. In other words, the raw data can be recovered from the following expression: $S_{\text {raw }}(t)=\sum_{i=1}^{N} C_{i}(t)+$ $\mathrm{R}_{N}(t)$.

\subsection{Entropic analysis}

Because the adaptive nature of EMD is likely to distribute signals that contain no information that is relevant to the underlying functions into IMFs, it was crucial to exclude such IMFs before we properly interpreted the decomposition results. Wu and Huang (2004) proposed a solution in which a hypothesis test method is developed to determine the statistical significance of information content in IMFs by considering the mean energy. However, because their method only accounted for the macroscopic properties of the signals, mean energy and mean period, the detailed information of the IMFs was not revealed completely, and the interpretation may have been limited. In this study, we introduced an entropic analysis to resolve this issue (Tseng and Lee, 2010). The idea was to consider all the amplitudes of the IMFs and to determine whether a probability distribution of the amplitudes of an IMF from signals is identical to the probability distribution of noise with the same energy density. 
The method consists of two steps. First, we needed to determine the probability distributions of amplitudes of IMFs from real signal and noise. Second, we evaluated the relative entropy or Kullback-Leibler distance of these two distributions to determine their difference (Tseng, 2006; Chen et al., 2007). For the probability distributions from real signals, we simply utilized normalized histograms of amplitudes $p_{i}^{\text {real }}(t)$. We then addressed the following question: Given the energy of this probability distribution, what is the preferred probability distribution of amplitudes of IMFs decomposed from noise? Because the energy of normalized white noise is given by $E_{i}=\sum_{t=1}^{N} C_{i}^{2}(t) / N$, the method of maximum entropy can be applied to determine the probability distribution from noise (Jaynes, 1957). Therefore, given the energy of an IMF from normalized white noise, the probability distribution of an amplitude in that IMF is given by $p_{i}^{\text {noise }}(t)=e^{-\lambda C_{i}^{2}(t)} / Z$, where $Z=\sum_{t=1}^{N} e^{-\lambda C_{i}^{2}(t)}$ and $\lambda$ is a Lagrangian multiplier to be determined using $E_{i}$. The amplitudes of the IMFs decomposed from white noise are then normally distributed, which is also confirmed from $\mathrm{Wu}$ and Huang's numerical studies (Wu and Huang, 2004). Finally, we determined the difference between the probability distributions $p_{i}^{\text {real }}(t)$ and $p_{i}^{\text {noise }}(t)$ by calculating the relative entropy $S\left(p_{i}^{\text {real }}, p_{i}^{\text {noise }}\right)=$ $-\sum_{t=1}^{N} p_{i}^{\text {real }}(t) \log p_{i}^{\text {real }}(t) / p_{i}^{\text {noise }}(t)$. When $p_{i}^{\text {real }}(t)$ was identical to $p_{i}^{\text {noise }}(t)$, i.e. $p_{i}^{\text {real }}(t)$ was generated from noise, then $S\left(p_{i}^{\text {real }}, p_{i}^{\text {noise }}\right)$ was maximized and equaled zero.

\subsection{Spectral analysis}

Spectral analysis using the FT is a tool for determining the frequency-domain representation of a time-domain signal and examining a function from the perspectives of both the time and frequency domains. The discrete Fourier transform (DFT) is used to determine the frequency content of finite-length and analog/digital signals, and the fast Fourier transform (FFT) is an efficient method of calculating the DFT (Brigham, 1988). We calculated the power spectral density (PSD) of IMFs using a periodogram associated with the FFT (Stoica and Moses, 1997), which allowed us to examine the periodicities of the IMFs and to identify the underlying functions of the triggering sources.

\subsection{Stacking}

In addition to the techniques described above, we also applied the stacking technique to suppress and smooth the raw data, which were usually contaminated by irregular noise. Irregular noise is often random and thus can be suppressed by stacking the raw data. Stacking can increase the S/N ratio and enhance the inherent periodic signals. The stacking technique used here is based on the in-phase phenomenon of the theoretical Earth tides. The time series of the theoretical Earth tides are in phase every 1462 days, resulting in a periodic variation with a period of 1462 days. Hence, stacking the daily earthquake numbers over time spans of 1462 days can enhance the weakly periodic signals (if any) and also suppress irregular noise.

\subsection{Significance test}

We utilized a stochastic test to investigate the statistical significance of the observed tidal triggering effect. Because time was the key factor in our investigations here, we uniformly randomized the occurrence times of earthquakes with $M_{\mathrm{L}} \geqq M_{\mathrm{c}}=3.0$ in three types of earthquake catalogs (non-declustered, R-declustered, and GK-declustered cata$\operatorname{logs}$ ) and analyzed the randomized data using the procedure mentioned above. We repeated the randomized realization 200 times to obtain sufficient statistical samples. To examine whether the PSDs of the IMFs that were decomposed from real data were statistically significant, we then calculated the mean and standard deviation of the PSDs of the IMFs that were decomposed from randomized data. If the PSDs of tne IMFs of the real data are within those obtained from the randomized data with a $95 \%$ confidence level, those PSDs obtained from the real data were considered to lack statistical significance. Such an approach allowed us to examine whether the peaks of the tidal triggering mechanism were statistically significant.

\section{Results}

Figure 2a shows the time series of daily earthquakes with $M_{\mathrm{L}} \geqq 3.0$ in Taiwan. The numerous spikes observed in this plot are associated with large earthquake sequences. The highest spike (E9) is around 20 September 1999, which is the date of the $M=7.3$ Chi-Chi earthquake (Ma et al., 1999). The E1 spike represents the $M=6.8$ Lanhsu earthquake on 23 July 1978; E2 is the $M=6.8$ Hualien earthquake on 23 December 1978; E3 is the $M=6.2$ Hualien earthquake on 20 May 1986; E4 is the $M=7.8$ Hualien earthquake on 14 November 1986; and E5 is the $M=6.7$ Hualien earthquake on 13 December 1990. E6 represents the $M=6.7$ Hualien earthquake on 24 May 1994; E7 is the $M=6.3$ Nanao earthquake on 5 June 1994; E8 is the $M=7.1$ Lanyu on 5 September 1996; E10 is the $M=7.1$ Hualien earthquake on 31 March 2002; E11 is the $M=6.8$ Chengkung earthquake on 10 December 2003; and E12 is the $M=7.0$ Hengchun earthquake on 26 December 2006. These sequences were reported in Wang (1998), Wu et al. (2008) and on the CWB website (http://www.cwb.gov.tw/ V7e/earthquake/). Figure 2a also shows the time series of the theoretical Earth tides calculated at the center of Taiwan. The theoretical Earth tides were computed from a model based on the HW95 tidal potential catalog (Hartmann and Wenzel, 1995; Wenzel, 1997). The catalog contains 12935 tidal waves with 19300 adjustable model coefficients caused by 


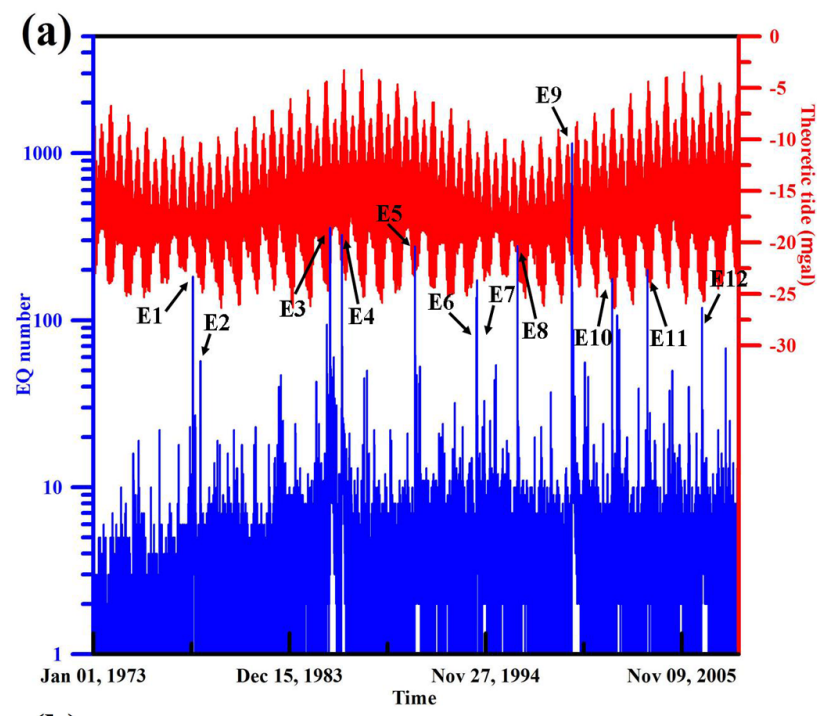

(b)

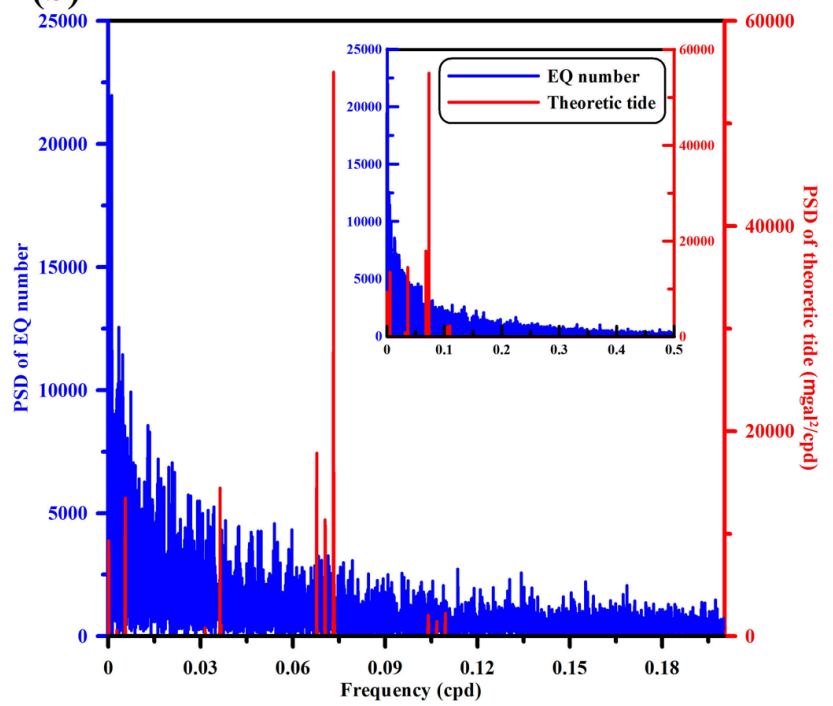

Fig. 2. (a) Time series of the number of daily earthquakes in Taiwan (blue) and theoretical Earth tides (red) calculated at the center of Taiwan; and (b) the Fourier power spectral density of time series of daily earthquakes and theoretical Earth tides. The symbols from E1 to E12 indicate large earthquakes in Taiwan.

the gravitational forces due to the sun, moon, and other celestial bodies. A periodic variation with a period of 1462 days can be found in the theoretical Earth tide.

Figure $2 b$ presents the Fourier PSD of time series of the daily earthquakes along with that of the theoretical Earth tides. The Fourier PSD of the time series of the daily earthquakes decreases with increasing frequency $(f)$ in units of cycles per day (cpd). However, the power density of the theoretical Earth tides is distributed at certain discrete frequencies and has a maximum amplitude of $\sim 55000 \mathrm{\mu gal}^{2} / \mathrm{cpd}$ at $f=\sim 0.073 \mathrm{cpd}$, which corresponds to a period of $\sim 13.7$ days. The other significant peaks are $\sim 14000 \mathrm{\mu gal}^{2} / \mathrm{cpd}$ at $f=\sim 0.036 \mathrm{cpd}(\sim 27.78$ days $)$ and $\sim 13000 \mathrm{\mu gal}^{2} / \mathrm{cpd}$ at $f=\sim 0.0055 \mathrm{cpd}(\sim 181.82$ days $)$.
Figure 3a shows the first six IMFs, i.e. IMF1 to IMF6. Because our goal was to investigate the effects of tidal triggering, of which the lunar tide (periods of 13.70 days and 27.78 days) is the largest, we focused on periods that ranged from 5 to 100 days, i.e. the frequency band from 0.01 to $0.2 \mathrm{cpd}$. Therefore, we focused our discussion on the first six IMFs extracted from the time series of daily earthquakes. As mentioned above, the strongest peak in the six IMFs, E9, is associated with the $M=7.3$ Chi-Chi earthquake on 20 September 1999. Peaks related to various large events are also found in these IMFs. To increase the $\mathrm{S} / \mathrm{N}$ ratio, we stacked the raw daily earthquake numbers based on theoretical Earth tides in Taiwan that are in phase every 1462 days (top panel of Fig. 3b). Note that five sets of theoretical Earth tides with phases of 1462 days with four wave envelopes are shown in this panel. The stacked time series of daily earthquake numbers are also plotted in the lower part of the first panel of Fig. 3b. Figure 3b also shows IMF1 through IMF6, which were extracted from the stacked time series of the daily earthquake numbers.

The Fourier PSDs of the IMFs of the non-stacked and stacked earthquake data from the non-declustered catalog are shown in Fig. 4a and b, respectively. The Fourier PSDs of IMF1-IMF6 are plotted from top to bottom. Note that these IMFs contain average frequencies in decreasing order. The first IMF, IMF1, indicates the maximum rate of change in amplitude and has the highest frequency content. The rate of change and frequency content of an IMF are generally lower than those of the previous IMF. These IMFs have remarkable individual frequency ranges that allow us to distinguish their physical meanings. Also plotted in dashed lines are the PSDs, at a $95 \%$ confidence level, of the IMFs decomposed from the randomized catalogs. Note that the PSD of the theoretical Earth tides is also superposed in each plot of Fig. 4 for comparison.

The Fourier PSDs of IMFs 3-5 of the non-stacked and stacked earthquake data from the R-declustered catalog are shown in Fig. 5a and b, and those of the non-stacked and stacked earthquake data from the GK-declustered catalog are shown in Fig. 5c and d. The PSDs at a $95 \%$ confidence level of the IMFs decomposed from the randomized catalogs and the PSD of the theoretical Earth tides are also plotted in Fig. 5. The PSDs in Fig. 5b and d share common features with coincident stronger peaks.

We listed the results of the entropy analysis in Table 1 , which shows the relative entropy values $(S)$ of each IMF obtained from both real and random earthquake data. The $S$ values of each IMF of the random earthquake data were calculated at a $95 \%$ confidence level from 200 sets of randomized earthquake catalogs. Cases with $S_{\text {real }}<S_{\text {rand }}$ indicate that the real IMF is unlikely to be generated from a hypothetical random process and has statistical significance. Most of the IMFs extracted from the real data have obvious statistical significance. 
(a)

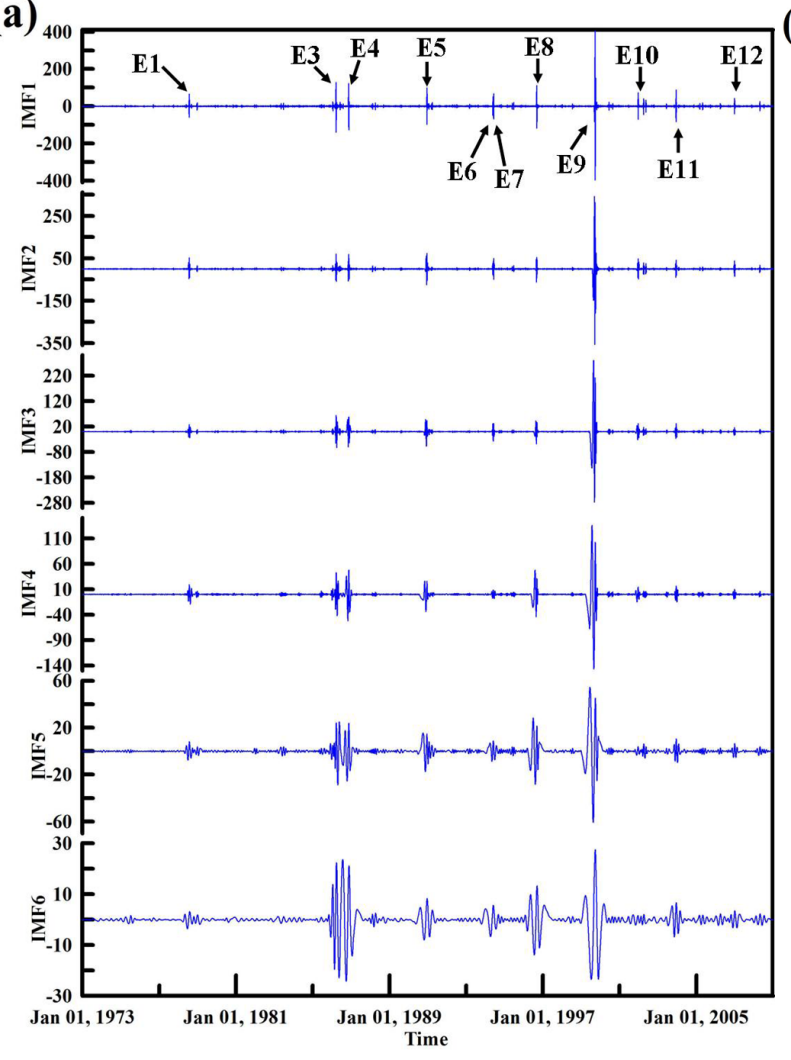

(b)

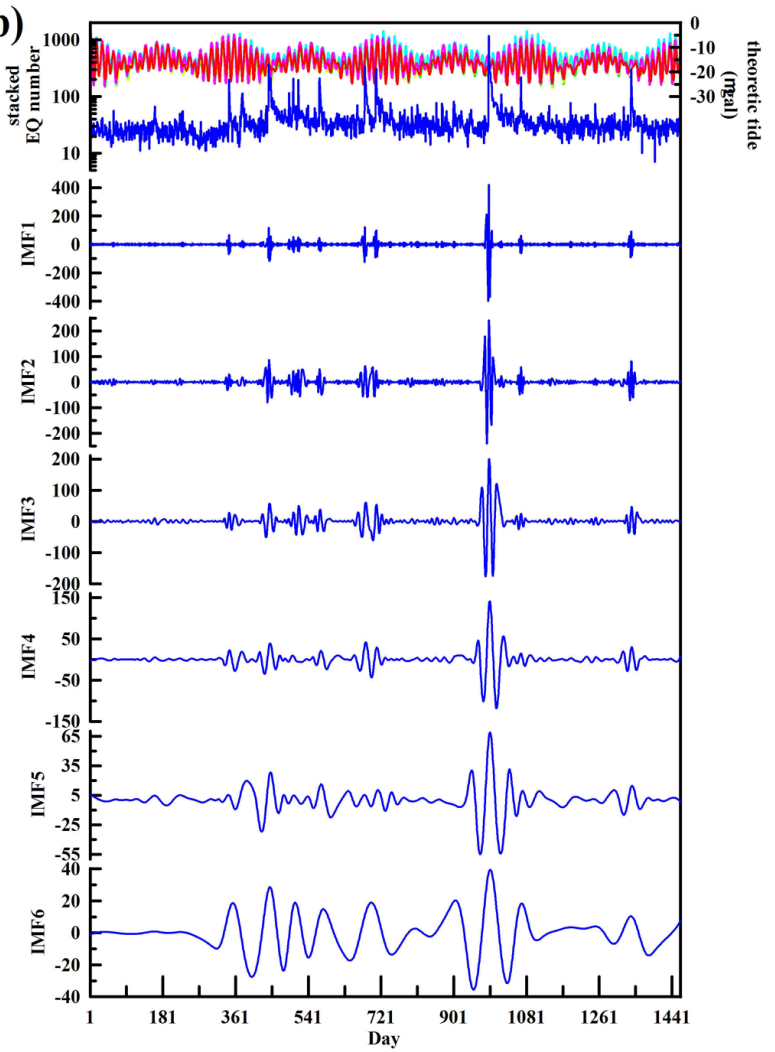

Fig. 3. (a) The first six IMFs (IMF1 to IMF6 from top to bottom) of non-stacked data of the number of daily earthquakes from the nondeclustered catalog in Taiwan. The symbols indicate the clear peaks in the IMFs that are associated with large earthquakes. (b) The top panel represents the temporal variations in theoretical Earth tides at the center of Taiwan and the stacked data of daily earthquake numbers from the non-declustered catalog. The upper part of the first panel shows five sets of Earth tides with a period of 1462 days. The second to seventh panels display the IMFs (IMF1 to IMF6) that were extracted from the stacked time series.

Table 1. The relative entropy values of the real data and the random data at a $95 \%$ confidence level.

\begin{tabular}{lccccc}
\hline & $\begin{array}{c}\text { Non-dec \& non-stack } \\
\text { catalog/random }\end{array}$ & $\begin{array}{c}\text { Non-dec \& stack } \\
\text { catalog/random }\end{array}$ & $\begin{array}{c}\text { R-dec \& } \\
\text { non-stack catalog/random }\end{array}$ & $\begin{array}{c}\text { R-dec \& } \\
\text { stack catalog/random }\end{array}$ & $\begin{array}{c}\text { GK-dec \& } \\
\text { non-stack catalog/random }\end{array}$ \\
stack catalog/random \\
\hline IMF1 & $-1.162 /-0.062$ & $-0.991 /-0.108$ & $-0.105 /-0.061$ & $-0.102 /-0.112$ & $-0.037 /-0.057$ \\
IMF2 & $-1.800 /-0.008$ & $-0.916 /-0.054$ & $-0.159 /-0.006$ & $-0.132 /-0.052$ & $-0.022 /-0.007$ \\
IMF3 & $-1.991 /-0.007$ & $-0.953 /-0.056$ & $-0.235 /-0.007$ & $-0.074 /-0.058$ & $-0.042 /-0.008$ \\
IMF4 & $-1.619 /-0.008$ & $-0.852 /-0.068$ & $-0.158 /-0.009$ & $-0.090 /-0.066$ & $-0.038 /-0.009$ \\
IMF5 & $-1.187 /-0.013$ & $-0.536 /-0.089$ & $-0.466 /-0.010$ & $-0.116 /-0.084$ & $-0.037 /-0.011$ \\
IMF6 & $-0.975 /-0.018$ & $-0.273 /-0.121$ & $-0.213 /-0.026$ & $-0.262 /-0.115$ & $-0.079 /-0.059$ \\
\hline
\end{tabular}

\section{Discussion}

Figure 2a shows that the peaks associated with events E1E12 in the time series of the numbers of daily earthquake slightly appear to correspond to the low tide. These results appear to be consistent with studies by Wilcock (2001, 2009) and Tolstoy et al. (2002), who observed that microearthquakes occur preferentially at or near the days of low tide. However, there is no obvious correlation between the peaks at $f=\sim 0.036$ and/or $\sim 0.073 \mathrm{cpd}$ and the monthly and/or fortnightly tidal frequencies in the PSD of the daily earthquake numbers (Fig. 2b).
Due to the nonlinear and non-stationary nature of seismicity, the conventional Fourier power spectra could be contaminated by, for example, a large number of aftershocks (Telesca et al., 2009) and thus may not be appropriate for investigating the effect of tidal triggering. The occurrence of large earthquakes and their aftershocks can obviously increase the number of daily earthquakes, thus producing spikes in the time series (Fig. 2a). Based on the EMD, the IMFs of the nonstacked data of the daily earthquake numbers from the nondeclustered catalog (Fig. 3a) reveal several spikes associated with events E1-E12. Although the spikes associated with 
(a)

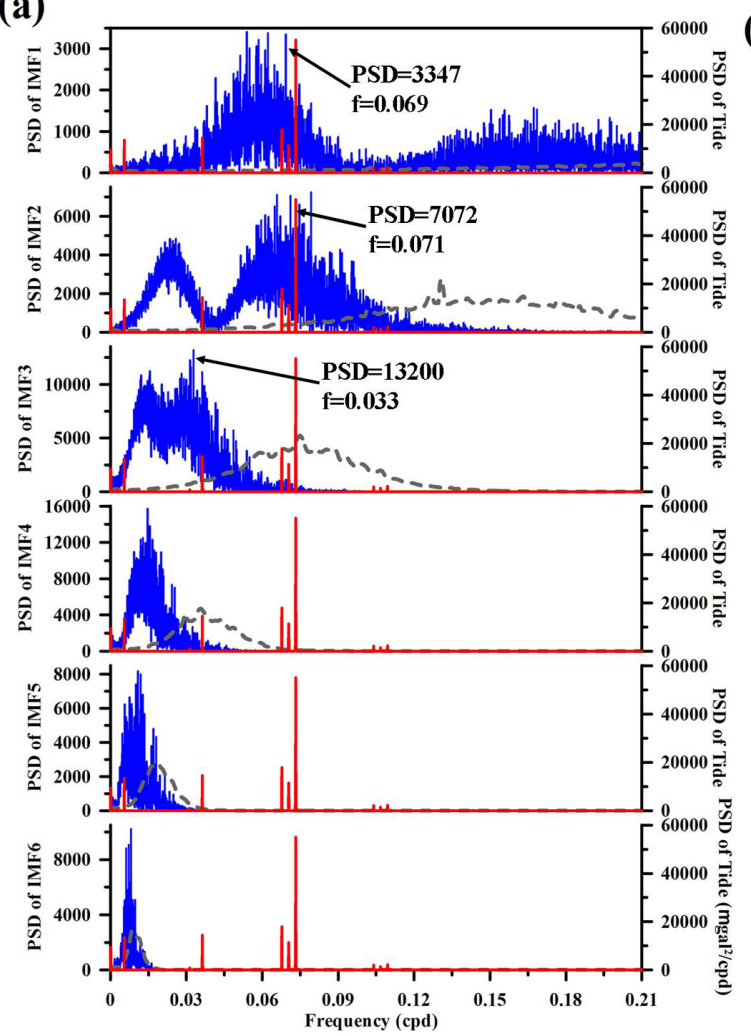

(b)

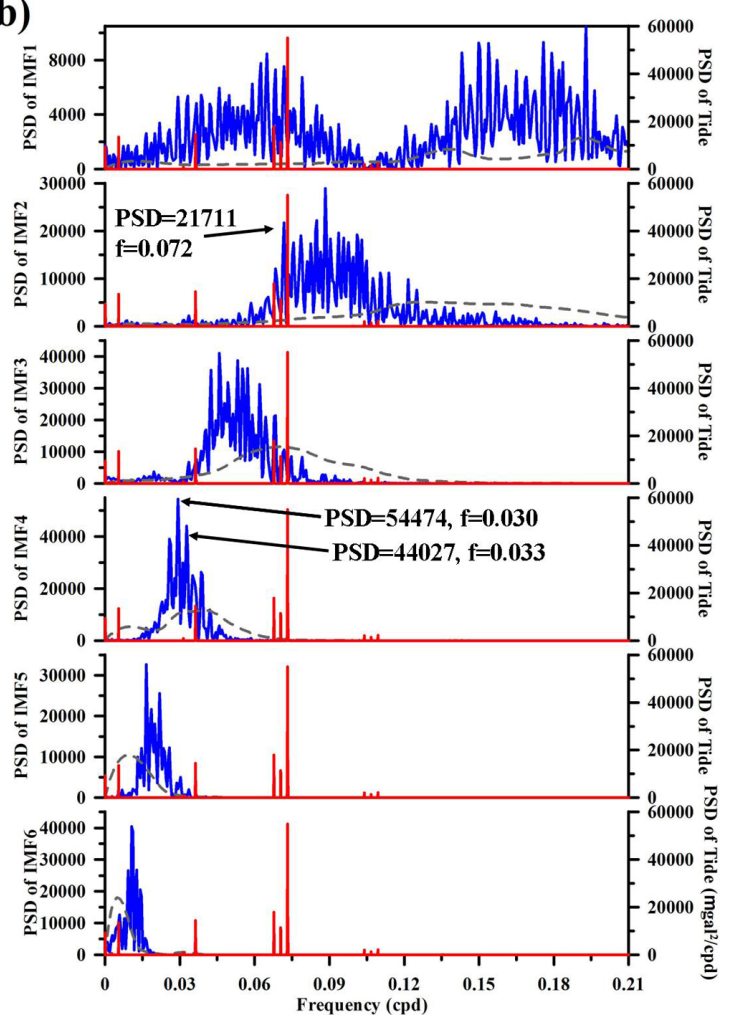

Fig. 4. The Fourier PSDs of the IMFs (IMF1 to IMF6 from top to bottom) that were extracted from (a) non-stacked and (b) stacked data of the non-declustered earthquake catalog. The red lines and gray lines represent the PSD of the Earth tides and the PSDs at a $95 \%$ confidence level from random earthquake catalogs, respectively.

those events are all identified in the six IMFs, those peak amplitudes gradually decrease from IMF1 to IMF6. The spikes will be decomposed into most of the IMFs with various frequency contents, hence diminishing the effect of the Fourier PSD of a spike (delta) function. The decrease of the high peak value by the EMD method aids the following Fourier spectral analysis.

The oscillation modes of the IMFs that were extracted from the stacked time series (Fig. 3b) can be described by harmonic functions with increasing periodicities from IMF1 to IMF6. Because stacking superposes the counts of daily earthquake numbers, the values of the IMFs from the stacked time series increase, thus amplifying the PSDs of the IMFs from the stacked time series. Note that the time scale in the stacked time series of daily earthquake numbers is 1462 days. The degree of clustering of aftershocks is reduced by stacking; hence, the degree of inherent regularity of earthquake occurrence would be increased, especially for small events.

The first column of Table 1 indicates that, based on the entropy analysis, IMFs 1-6, which were extracted from the non-stacked time series of the non-declustered catalog, are not generated from noise because $S_{\text {real }}<S_{\text {rand }}$. Additionally, the distributions of the real PSDs of the IMFs are different from those of the random PSDs at a 95\% confidence level
(Fig. 4a). Hence, the PSD of $\sim 3000$ at $\sim 0.069$ cpd (corresponding to $\sim 14.49$ days) in IMF1, the PSD of $\sim 7000$ at $\sim 0.071 \mathrm{cpd}$ (corresponding to $\sim 14.08$ days) in IMF2, and the PSD of $\sim 13000$ at $\sim 0.033 \mathrm{cpd}$ (corresponding to $\sim 30.30$ days) in IMF3 were likely caused by lunar tidal triggering. The second column of Table 1 indicates that IMFs 2 through 6 , which were extracted from the stacked time series of the non-declustered catalog, were not generated from noise. In addition, their PSDs are also different from those of the random PSDs at a 95\% confidence level (Fig. 4b). A comparison between Fig. $4 \mathrm{a}$ and $\mathrm{b}$ shows that the amplitudes of the PSDs of the IMFs from the stacked data at the lunar tidal frequencies are about twice as large as those from the non-stacked data, which was caused by the stacking method constructively amplifying the in-phase signals. This indirectly suggests the existence of the lunar tidal triggering mechanism.

Consequently, the existence of the peak PSD values of approximately 0.036 and $0.073 \mathrm{cpd}$ in the PSDs of IMFs $1-3$ for the non-stacked data and in those of IMFs 2-4 for the stacked data suggests that the lunar tidal force is likely one of the mechanisms that triggers earthquakes. The lunar tidal force may not be able to be identified from the raw data of daily earthquake numbers (Fig. 2b) because of the weak 
(a)

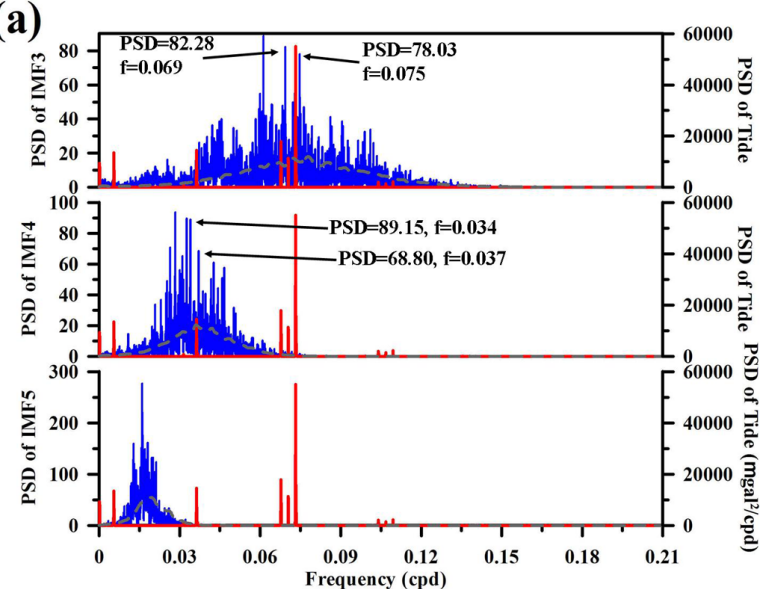

(c)

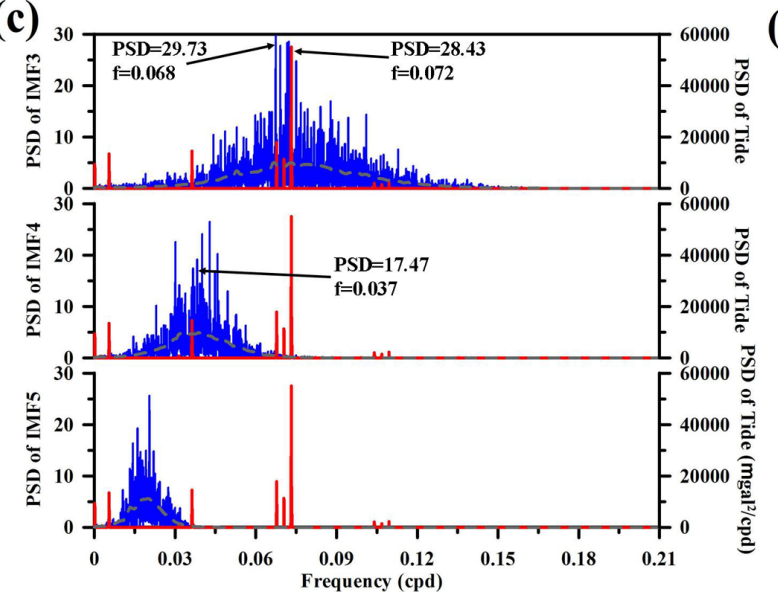

(b)

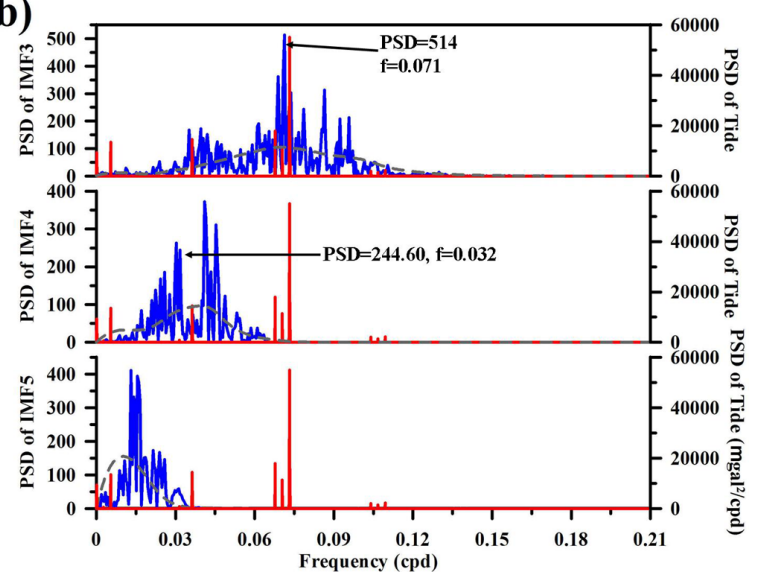

(d)

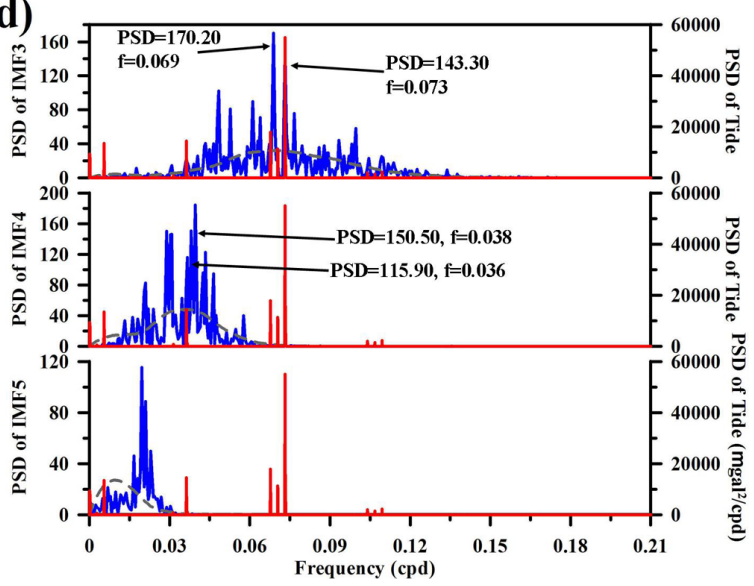

Fig. 5. The Fourier PSDs of IMFs (IMF3 to IMF5 from top to bottom) extracted from (a) non-stacked and (b) stacked earthquake data from the Resenberg declustered catalog, and those from (c) non-stacked and (d) stacked earthquake data from the Gardner and Knopoff declustered catalog. The PSD of theoretical Earth tides calculated at the center of Taiwan are displayed by the red line segments, and the PSDs at a $95 \%$ confidence level are depicted by the dashed lines.

periodicity of the signals due to contamination by strong background seismicity.

Similarly, there are relatively strong power densities in the PSDs of the IMFs from the non-stacked data of the R-declustered catalog (Fig. 5a), i.e. PSD $=82.28$ at $f=0.069 \mathrm{cpd}(\sim 14.49$ days $)$ and $\mathrm{PSD}=78.03$ at $f=$ $0.075 \mathrm{cpd}(\sim 13.33$ days $)$ in IMF3 and PSD $=89.15$ at $f=$ $0.034 \mathrm{cpd}(\sim 29.41$ days $)$ and $\mathrm{PSD}=68.80$ at $f=0.037 \mathrm{cpd}$ ( $\sim 27.03$ days) in IMF4. After stacking (Fig. $5 b$ ), PSD $=514$ at $f=0.071 \mathrm{cpd}(\sim 14.08$ days $)$ in IMF3 and $\mathrm{PSD}=244.60$ at $f=0.032 \mathrm{cpd}(\sim 31.25$ days $)$ in IMF4 have stronger peaks. Those peak PSD values at lunar tidal frequencies and their IMFs are also identified in the entropy analysis and the significance test. The power densities at the lunar tidal frequencies of the stacked data are also greater than those of the non-stacked data. Hence, the lunar tidal triggering mechanism is also clearly shown in the R-declustered catalog. On the other hand, the most noticeable peaks in the PSDs of the IMFs from the non-stacked GK-declustered catalog
(Fig. 5c) are $\mathrm{PSD}=29.73$ at $f=0.068 \mathrm{cpd}(\sim 14.71$ days $)$ and $\mathrm{PSD}=28.43$ at $f=0.072 \mathrm{cpd}(\sim 13.89$ days $)$ in IMF3 and $\mathrm{PSD}=17.47$ at $f=0.037 \mathrm{cpd}(\sim 27.03$ days $)$ in IMF4. After stacking, the peaks are PSD $=170.20$ at $f=0.069 \mathrm{cpd}(\sim 14.49$ days $)$ and $\mathrm{PSD}=143.30$ at $f=$ $0.073 \mathrm{cpd}(\sim 13.70$ days $)$ in IMF3, and PSD $=115.90$ at $f=$ $0.036 \mathrm{cpd}(\sim 27.78$ days $)$ and $\mathrm{PSD}=150.50$ at $f=0.038 \mathrm{cpd}$ ( $\sim 26.32$ days) in IMF4, whose peak values are at least three times greater than those of the non-stacked data. Those peak PSD values at lunar tidal frequencies and their IMFs are also interpreted as tidal signals after the entropy analysis and significance test.

Both types of declustered results confirm the existence of a tidal triggering effect. It is reasonable that the tidal effect still exists in the declustered catalog because the strong aftershock effect is transient in the daily earthquake number time series. The EMD-based analysis may ignore the transient mechanisms. Our studies here are consistent with previous studies that have proposed fortnightly tidal triggering 
(e.g. Allen, 1936; Souchay and Stavinschi, 1999; Lin et al., 2003). Although Hartzell and Heaton (1989) claimed that fortnightly periodicity is not significant in Southern California and world-wide earthquake catalogs, we argue that the statistical significances could be biased by earthquake data selection. In summary, the apparent inconsistency regarding the tidal triggering issue in the literature could be a result of the sample populations, analytical methods, earthquake magnitude cutoffs and, possibly, tectonic setting. In this work, we have proposed a new method that can analyze nonlinear and/or non-stationary data and likely detect the tidal effect more precisely than other methods.

\section{Conclusions}

The EMD method was applied with spectral analysis to earthquake data in the Taiwan region to investigate the effect of tidal triggering on earthquake occurrence. The proposed method consists of source separation by EMD, entropy analysis, Fourier power spectral analysis of IMFs, data suppression and smoothing by stacking, and the statistical significance test. The results show that, for the Taiwan CWB earthquake catalog, the lunar tidal triggering effect was observed in IMFs 1-3, which were extracted from the non-stacked daily earthquake number time series of the non-declustered catalog, as well as in IMFs 2-4 from the stacked time series. In the R-declustered catalog, the lunar tidal effect was observed in IMFs 3-4 from the non-stacked time series as well as in IMFs 3-4 from the stacked time series. In the GK-declustered catalog, the lunar tidal effect was found in IMFs 3-4 from the non-stacked time series as well as in IMFs 3-4 from the stacked time series. Therefore, we identified the tidal triggering effect in both the non-declustered and declustered catalogs. All of the results are consistent with the peaks at $f=0.036 \mathrm{cpd}(\sim 27.78$ days $)$ and $f=0.073 \mathrm{cpd}$ ( $\sim 13.70$ days) in the PSD of the theoretical Earth tides. In addition, the peak PSD values are statistically significant because they are greater than the random PSD values. In conclusion, the lunar tidal force with a period of $\sim 14$ days and $\sim 28$ days likely influences earthquake occurrence.

As a final remark, we emphasize that other methods could be also used to evaluate the tidal effect in various geophysical signals in addition to the EMD-based approach proposed here (Huang and Liu, 2006; Tsolis and Xenos, 2010). One is the BAYTAP method, which has been used to analyze geoelectric potential data (Huang and Liu, 2006). We are interested in whether we can detect the tidal components in seismicity data using the BAYTAP method and will consider this in our future work.

Acknowledgements. CCC is grateful for research support from the National Science Council (ROC) and the Graduate Institute of Geophysics, National Central University (ROC). We are indebted to two anonymous reviewers for their helpful comments.
Edited by: M. E. Contadakis

Reviewed by: T. D. X. Xenos and another anonymous referee

\section{References}

Allen, M. W.: The lunar triggering effect on earthquakes in Southern California, Bull. Seism. Soc. Am., 26, 147-157, 1936.

Beeler, N. M. and Lockner, D. A.: Why earthquakes correlate weakly with the solid Earth tides: Effects of periodic stress on the rate and probability of earthquake occurrence, J. Geophys. Res., 108(B8), 2391, doi:10.1029/2001JB001518, 2003.

Brigham, E. O.: The Fast Fourier Transform and Its Application, 1st Edn., ISBN-10: 0133075052, 1988.

Caticha, A.: Bayesian Inference and Maximum Entropy Methods in Science and Engineering, edited by: Erickson G. and Zhai Y., AIP, Melville, NY, USA, AIP Conf. Proc. 707, 75 pp., 2004.

Chen, C.-C., Tseng, C.-Y., and Dong, J.-J.: New Entropy-based Method for Variables Selection and Its Application to the Debrisflow Hazard Assessment, Eng. Geol., 94, 19-26, 2007.

Cochran, S. E., Vidale, J. E., and Tanaka, S.: Earth tides can trigger shallow thrust fault earthquakes, Science, 306, 1164-1166, doi:10.1126/science.1103961, 2004.

Davison, C.: The diurnal periodicity of earthquakes, J. Geol., 42, 449-468, 1934.

Fischer, T., Kalenda, P., and Skalský, L.: Weak tidal correlation of NW- Bohemia/Vogtland earthquake swarms, Tectonophysics, 424, 259-269, 2006.

Gardner, J. K. and Knopoff, L.: Is the sequence of earthquakes in Southern California, with aftershocks removed, Poissonian, Bull. Seismol. Soc. Am., 64, 1363-1367, 1974.

Gutenberg, B. and Richter, C. F.: Seismicity of the Earth and Associated Phenomena, 2nd Edn., Princeton Univ. Press, Princeton, New Jersey, 310 pp., 1954.

Hartmann, T. and Wenzel, H. G.: The HW95 tidal potential catalogue, Geophys. Res. Lett., 22, 3553-3556, 1995.

Hartzel, S. and Heaton, T.: The fortnightly tide and the tidal triggering of earthquakes, Bull. Seismol. Soc. Am., 79, 1282-1286, 1989.

Heaton, T. H.: Tidal triggering of earthquakes, Bull. Seismol. Soc. Am., 72, 2181-2200, 1982.

Huang, Q.: Search for reliable precursors: A case study of the seismic quiescence of the 2000 western Tottori prefecture earthquake, J. Geophys. Res., 111, B04301, doi:10.1029/2005JB003982, 2006.

Huang, Q.: Seismicity changes prior to the Ms8.0 Wenchuan earthquake in Sichuan, China, Geophys. Res. Lett., 35, L23308, doi:10.1029/2008GL036270, 2008.

Huang, N.-E., Shen, Z., Long, S.-R., Wu, M.-C., Shih, E.-H., Zheng, Q., Yen, N.-C., Tung, C.-C., and Liu, H.-H.: The empirical mode decomposition method and the Hilbert spectrum for nonstationary time series analysis, Proc. R. Soc. Lond. A, 454, 903995, 1998.

Huang, Q. and Liu, T.: Earthquakes and tide response of geoelectric potential field at the Niijima station, Chinese J. Geophys., 49, 1745-1754, 2006.

Jaynes E. T.: Information theory and statistical mechanics, Phy. Rev., 106, 620-630, 1957.

Kanamori, H. and Anderson, D. L.: Theoretical basis of some empirical relations in seismology, Bull. Seism. Soc. Am., 65, 1073- 
1095, 1975.

Keilis-Borok, V. I.: The lithosphere of the Earth as a nonlinear system with implications for earthquake prediction, Rev. Geophys., 28, 19-34, 1990.

Kilston, S. and Knopoff, L.: Lunar-solar periodicities of large earthquakes in southern California, Nature, 304, 21-25, 1983.

Kizhner, S., Flatley, T. P., Huang, N.-E., Blank, K., Conwell, E., and Smith, E.: On the Hilbert-Huang Transform data processing system development, Proc. IEEE Aerospace Conf., 1961-1979, 2004.

Knopoff, L.: Earth tides as a triggering mechanism for earthquakes, Bull. Seismol. Soc. Am., 54, 1865-1870, 1964.

Lambert, A., Kao, H., Rogers, G., and Courtier, N.: Correlation of tremor activity with tidal stress in the northern Cascadia subduction zone, J. Geophys. Res., 114, B00A08, doi:10.1029/2008JB006038, 2009.

Lin, C.-H., Yeh, Y.-H., Chen, Y.-I., Liu, J.-Y., and Chen, K.-J.: Earthquake clustering relative to lunar phases in Taiwan, Terr. Atmos. Ocean. Sci., 14, 289-298, 2003.

Ma, K. F., Lee, C. T., Tsai, Y. B., Shin, T. C., and Mori, J.: The Chi-Chi, Taiwan earthquake: Large surface displacements on an inland thrust fault, Eos, Transaction, AGU, 80, 605-611, 1999.

Métivier, L., Viron, O., Conrad, C. P., Renault, S., Diament, M., and Patau, M.: Evidence of earthquake triggering by the solid earth tides, Earth Planet. Sci. Lett., 278, 370-375, 2009.

Nasu, N., Kishinouye, F., and Kodaira, T.: Recent seismicity activities in the Idu Peninsula, Bull. Earthquake Res. Inst., 9, 22-35, 1931.

Palumbo, A.: Lunar and solar tidal components in the occurrence of earthquakes in Italy, Geophys. J. R. Astr. Soc., 84, 93-99, 1986.

Rai, V. K. and Mohanty, A. R.: Bearing fault diagnosis using FFT of intrinsic mode functions in Hilbert-Huang transform, Mech. Syst. Signal Pr., 21, 2607-2615, 2007.

Resenberg, P.: Second-order moment of Central California seismicity, 1969-1982, J. Geophys. Res., 90, 5479-5495, 1985.

Shin, T.-C.: Some implications of Taiwan tectonic features from the data collected by the Central Weather Bureau Seismic Network, Meteorol. Bull., CWB, 38, 23-48, 1992 (in Chinese).

Simpson, J. F.: Earth tides as a triggering mechanism for earthquakes, Earth Planet. Sci. Lett., 2, 473-478, 1967.

Souchay, J. and Stavinschi, M.: Study of the correlations between long-periodic terrestrial tides and occurrence of earthquakes in the Vrancea stie, Earth Moon Planets, 77, 105-124, 1999.

Stetson, H. T.: The correlation of deep-focus earthquakes with lunar hour angle and declination, Science, 82, 523-524, doi:10.1126/science.82.2135.523, 1935.

Stoica, P. and Moses, R. L.: Introduction to Spectral Analysis, Prentice-Hall, 24-26, 1997.

Tanaka, S.: Tidal triggering of earthquakes precursory to the recent Sumatra megathrust earthquakes of 26 December 2004 (Mw 9.0), 28 March 2005 (Mw 8.6), and 12 September 2007 (Mw 8.5), Geophys. Res. Lett., 37, L02301, doi:10.1029/2009GL041581, 2010.

Tanaka, S., Ohtake, M., and Sato, H.: Evidence for tidal triggering of earthquakes as revealed from statistical analysis of global data, J. Geophys. Res., 107, 2211, doi:10.1029/2001JB001577, 2002.

Tanaka, S., Ohtake, M., and Sato, H.: Tidal triggering of earthquakes in Japan related to the regional tectonic stress, Earth Planets Space, 56, 511-515, 2004.
Telesca, L., Chen, C.-C., and Lee, Y.-T.: Scaling behaviour in temporal fluctuations of crustal seismicity in Taiwan, Nat. Hazards Earth Syst. Sci., 9, 2067-2071, doi:10.5194/nhess-9-2067-2009, 2009.

Tolsoty, M., Vernon, F. L., Orcutt, J. A., and Wyatt, F. K.: Breathing of the seafloor: Tidal correlation of seismicity, Geology, 30, 503506, 2002.

Tsai, Y.-B., Teng, T.-L., Chiu, J.-M., and Liu, H.-L.: Tectonic implications of the seismicity in the Taiwan region, Mem. Geol. Soc. China, 2, 13-41, 1977.

Tseng, C.-Y.: Entropic Criterion for Model Selection, Physica A, 370, 530-538, 2006.

Tseng, C.-Y. and Lee, H.-C.: Entropic Interpretation of Empirical Mode Decomposition and Its Applications in Signal Processing, Adv. Adapt. Data Anal., 2, 429-449, 2010.

Tsolis, G. S. and Xenos, T. D.: A qualitative study of the seismoionospheric precursors prior to the 6 April 2009 earthquake in L'Aquila, Italy, Nat. Hazards Earth Syst. Sci., 10, 133-137, doi:10.5194/nhess-10-133-2010, 2010.

Tsuruoka, H., Ohtake, M., and Sato, H.: Statistical test of the tidal triggering of earthquakes: Contribution of the ocean tide loading effect, Geophys. J. Int., 122, 183-194, 1995.

Vidale, J. E., Agnew, D. C., Johnston, M. J. S., and Oppenheimer, D. H.: Absence of earthquake correlation with Earth tides: An indication of high preseismic fault stress rate, J. Geophys. Res., 103, 24567-24572, 1998.

Wang, J.-H.: b values of shallow earthquakes in Taiwan, Bull. Seism. Soc. Am., 78, 1243-1254, 1988.

Wang, J.-H.: The Taiwan Telemetered Seismographic Network, Phys. Earth Planet. Inter., 58, 9-18, 1989.

Wang, J.-H.: Studies of earthquake seismology in Taiwan during the 1897-1996 period, J. Geol. Soc. China, 41, 291-336, 1998.

Wang, J.-H. and Kuo, C.-H.: On the frequency distribution of interoccurrence times of earthquakes, J. Seismol., 2, 351-358, 1998.

Wenzel, H. G.: Tide-generating potential for the Earth, Lect. Notes Earth Sci., 66, 9-26, 1997.

Wiemer, S. and Wyss, M.: Minimum magnitude of completeness in earthquake catalogs: Examples from Alaska, the western United States, and Japan, Bull. Seism. Soc. Am., 90, 859-869, 2000.

Wilcock, W. S. D.: Tidal triggering of microearthquakes on the Juan de Fuca Ridge, Geophys. Res. Lett., 28, 3999-4002, 2001.

Wilcock, W. S. D.: Tidal triggering of earthquakes in the Northeast Pacific Ocean, Geophys. J. Int., 179, 1055-1070, 2009.

Wu, F. T.: Recent tectonics of Taiwan, J. Phys. Earth, 2, S265-S299, 1978.

Wu, Y.-M., Chen, C.-C., Zhao, L., and Chang, C.-H.: Seismicity characteristics before the 2003 Chengkung, Taiwan, earthquake, Tectonophysics, 457, 177-182, 2008.

Wu, Y.-M. and Chen, C.-C.: Seismic reversal pattern for the 1999 Chi-Chi, Taiwan, MW 7.6 earthquake, Tectonophysics, 429, 125-122, 2007.

Wu, Z.-H. and Huang, N.-E.: A study of the characteristics of white noise using the empirical mode decomposition method, Proc. R. Soc. Lond. A, 460, 1597-1611, 2004. 